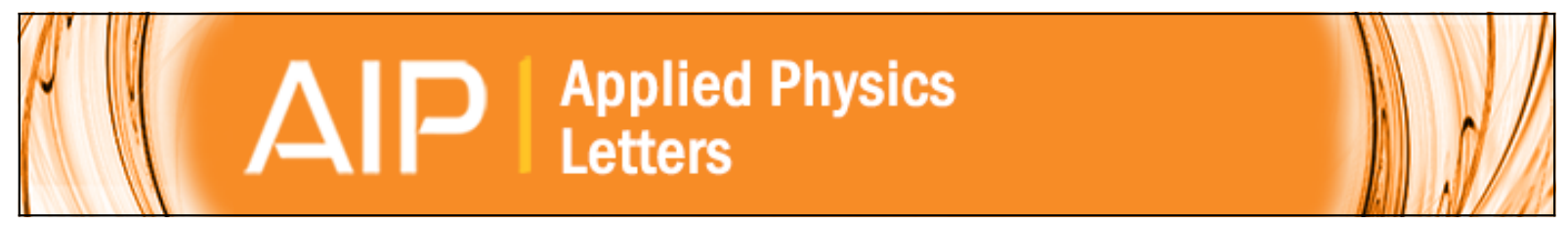

On the determination of internal optical mode loss of semiconductor lasers

P. M. Smowton and P. Blood

Citation: Applied Physics Letters 70, 2365 (1997); doi: 10.1063/1.118875

View online: http://dx.doi.org/10.1063/1.118875

View Table of Contents: http://scitation.aip.org/content/aip/journal/apl/70/18?ver=pdfcov

Published by the AIP Publishing 


\title{
On the determination of internal optical mode loss of semiconductor lasers
}

\author{
P. M. Smowton ${ }^{\mathrm{a})}$ and P. Blood \\ Department of Physics and Astronomy, University of Wales Cardiff, Cardiff CF2 3YB, United Kingdom
}

(Received 31 January 1997; accepted for publication 9 March 1997)

\begin{abstract}
Use is made of a numerical simulation of the light-current characteristic to examine the errors which may arise in the determination of the optical mode loss $\left(\alpha_{i}\right)$ from the cavity length dependence of the external differential efficiency $\left(\eta_{\mathrm{ext}}^{d}\right)$. In particular, we focus on the effects of incomplete Fermi level pinning and carrier leakage, and show that $\alpha_{i}$ can only be determined correctly if $\eta_{\mathrm{ext}}^{d}$ is determined under conditions where it is invariant both with current level and temperature. (C) 1997 American Institute of Physics. [S0003-6951(97)03318-4]
\end{abstract}

It is common to measure the internal optical mode loss $\left(\alpha_{i}\right)$ and internal differential quantum efficiency $\left(\eta_{o}^{d}\right)$ of semiconductor lasers by analysis of external differential efficiency $\left(\eta_{\text {ext }}^{d}\right)$ as a function of device length $\left(L_{c}\right)$, with $\eta_{\text {ext }}^{d}\left(L_{c}\right)$ measured as the slope of the power output per facet $\left(P_{\text {ext }}\right)$ as a function of current $(I)$ above threshold. In the accepted method, ${ }^{1} \alpha_{i}$ is determined from the slope of a plot of $\left(\eta_{\text {ext }}^{d}\right)^{-1}$ vs $L_{c}$, and $\eta_{o}^{d}$ from the intercept according to the relation

$$
\frac{1}{\eta_{\mathrm{ext}}^{d}}=\frac{1}{\eta_{o}^{d}}\left(1+\frac{\alpha_{i} L_{c}}{\ln (1 / R)}\right)
$$

where $R$ is the facet reflectivity. The central assumption of this analysis is that $\eta_{o}^{d}$ and $\alpha_{i}$ are independent of cavity length. Although an alternative technique has been proposed ${ }^{2}$ to determine $\alpha_{i}$ the approach of Equation (1) continues in widespread use.

It is commonly assumed that $\eta_{o}^{d}$ should have a value of unity above threshold, although the values derived from Equation (1) are often significantly less than this. We have shown that this is due to the failure of the Fermi levels to pin at threshold throughout the whole structure and that under certain circumstances $\eta_{o}^{d}$ is dependent upon cavity length. ${ }^{3}$ In the light of these findings, the purpose of this letter is to determine the conditions under which Equation (1) is a valid means for determination of $\alpha_{i}$ by numerical simulation of the light-current characteristic.

We have described the internal differential efficiency in terms of three components: ${ }^{3}$

$$
\eta_{o}^{d}=\eta_{s}^{d} \eta_{i}^{d} \eta_{r}^{d}
$$

The term $\eta_{s}^{d}$ is associated with lateral current spreading and it is generally thought that in broad area devices changes in current spreading and filamentation above threshold are associated with variations in the measured external differential efficiency and we believe that such changes often cause scatter in the results. In the presence of current spreading we have shown that $\eta_{s}^{d}$ is less than unity, although there is no evidence that $\eta_{s}^{d}$ is length dependent ${ }^{3}$ so the $\alpha_{i}$ analysis remains valid. The term $\eta_{i}^{d}$ is the injection efficiency representing the fraction of increase in current entering the active area of the device that results in recombination within the quantum well. It is less than unity if the Fermi levels do not pin outside the quantum well, and it represents current asso- ciated with recombination in the barrier and drift and diffusion through the cladding layers. We have shown that $\eta_{i}^{d}$ is extremely sensitive to quasi-Fermi level position and is therefore temperature and length dependent. ${ }^{3}$ Finally $\eta_{r}^{d}$ is the differential radiative efficiency of the quantum well itself and describes the fraction of the increase in current entering the well which results in an increase in the stimulated emission. We show below that $\eta_{r}^{d}$ is unity and independent of length providing data is taken over the appropriate current range above threshold. In what follows we focus on the effect of $\eta_{i}^{d}$ and $\eta_{r}^{d}$ on the $\alpha_{i}$ analysis.

To investigate the fundamental limitations in the use of Equation (1) we adopted a model of the output power and drive current based on the single mode rate equations. ${ }^{4}$ The power output from a single facet is given by ${ }^{5}$

$$
P_{\text {ext }}(W)=\frac{h c \alpha_{m} \beta_{\text {spon }} J_{\text {spon }} A}{2 \lambda e\left(\alpha_{m}+\alpha_{i}-\Gamma g\right)},
$$

where $\alpha_{m}$ is the mirror loss, calculated for $R=0.3, \beta_{\text {spon }}$ $=10^{-3}$ is the proportion of spontaneous emission emitted into the lasing mode; $J_{\text {spon }}$, the current density resulting from spontaneous recombination in the well; $A$ is the device area (length $\times$ width of $50 \mu \mathrm{m}$ ); $\Gamma=0.242$ is the optical confinement factor and $g$ the material gain.

The total drive current is made up of nonradiative recombination, spontaneous recombination, and stimulated recombination within the active region, and leakage of carriers out of the active region ${ }^{5}$

$$
I(A)=\frac{n L A e}{\tau_{\mathrm{nr}}}+J_{\text {spon }} A+\frac{J_{\text {spon }} A \Gamma g \beta_{\text {spon }}}{\alpha_{m}+\alpha_{i}-\Gamma_{g}}+J_{L} A,
$$

where $n$ is the carrier population of the active region, $L$ is the active region width, $\tau_{\mathrm{nr}}$ is the nonradiative lifetime in the quantum well which is taken to be $3 \mathrm{~ns}$ independent of $n$. $J_{L}$ is the leakage current density and includes recombination in the barrier and drift and diffusion through the cladding layers, and is calculated analytically by a thermal equilibrium model from the quasi-Fermi level positions specified by the carrier density (Ref. 5, section 3.4). The leakage (of electrons) through the $(p-)$ cladding layer is given by ${ }^{5,6}$

$$
\begin{aligned}
J_{L c}= & e D_{n} n_{c}\left\{\left(\frac{1}{L_{n}^{2}}+\frac{1}{4 z^{2}}\right)^{1 / 2}\right. \\
& \left.\times \operatorname{coth}\left[\left(\frac{1}{L_{n}^{2}}+\frac{1}{4 z^{2}}\right)^{1 / 2} w_{c}\right]+\frac{1}{2 z}\right\},
\end{aligned}
$$

${ }^{a)}$ Electronic mail: smowtonpm@cf.ac.uk 


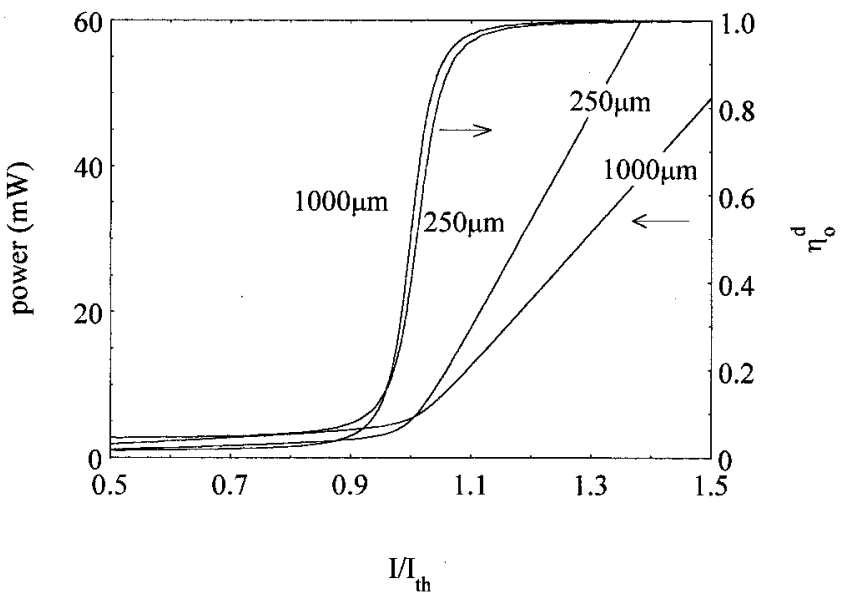

FIG. 1. Laser output power and overall internal differential efficiency derived from the slope of the $P_{\text {ext }}(I)$ characteristic vs current relative to threshold current for devices of length 250 and $1000 \mu \mathrm{m}$. The calculation only includes recombination within the quantum well itself $\left(\eta_{s}^{d}=\eta_{i}^{d}=1\right)$.

where $n_{c}$ is the electron density at the edge of the cladding layer (thickness $w_{c}$ and electrical conductivity $\sigma$ ), $L_{n}$ is the diffusion length, and $z$ is the drift length ${ }^{6}$ given by

$$
z=\frac{k T}{e}\left(\frac{\sigma}{J_{\text {active }}}\right),
$$

where $J_{\text {active }}$ is the majority carrier current flowing into the active region of well and barrier.

Optical power and current were calculated with the carrier density, $n$, as parameter using calculated gainspontaneous current and gain-carrier density characteristics for $68 \AA, \mathrm{Ga}_{.41} \mathrm{In}_{.59} \mathrm{P} /\left(\mathrm{Al}_{.5} \mathrm{Ga}_{.5}\right){ }_{.51} \mathrm{In}_{.49} \mathrm{P}$ quantum well lasers at $670 \mathrm{~nm} .^{7}$ Using the threshold condition for a specific cavity length we determined the threshold carrier density using the gain-carrier density characteristic. This is the limit of Eq. (3) where the power output, $P_{\text {ext }}$, becomes infinite. The carrier density was then reduced in increments leading to values of Fermi level positions, gain, and current, which were used to compute the optical power and drive current from Eqs. (3), (4), and (5), generating a light-current curve. We repeated the calculation for devices of length 250, 320, 450, 600,750 , and $1000 \mu \mathrm{m}$ with the other parameters remaining fixed. Throughout the input value of $\alpha_{i}$ was $10 \mathrm{~cm}^{-1}$. The parameter values chosen give a good description of $670 \mathrm{~nm}$ GaInP quantum well lasers. ${ }^{5}$

Figure 1 shows $P_{\text {ext }}$ versus current, relative to threshold current, for devices of length 250 and $1000 \mu \mathrm{m}$ at $300 \mathrm{~K}$ and with the leakage current $J_{L}=0$ in Eq. (4). We also show $\eta_{0}^{d}$ calculated from the local slope of the $P_{\text {ext }}(I)$ curve and corrected for the fraction of optical power lost from the cavity using Eq. (1). The figure shows, first, that $\eta_{0}^{d}$ does not immediately clamp above the threshold, even though the $P_{\text {ext }}(I)$ characteristic appears linear. Second, immediately above threshold the values of $\eta_{0}^{d}$ are different for different length devices so, even in the absence of any carrier leakage effects, the requirement that $\eta_{0}^{d}$ be independent of $L_{c}$ is not met till the current is more than $30 \%$ above threshold.

Figure 2 shows the effect of this behavior on the evaluation of $\alpha_{i}$. We have taken the local derivative of the

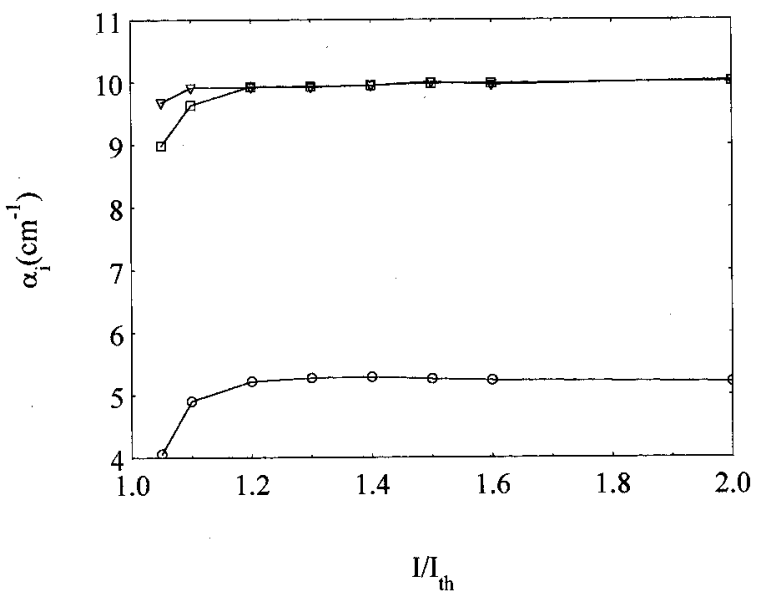

FIG. 2. Value of $\alpha_{i}$ derived from the $1 / \eta_{\text {ext }}^{d}$ vs $L_{c}$ analysis (input value $=10 \mathrm{~cm}^{-1}$ ) for three cases: (a) no loss of carriers from the quantum well ( $T=300 \mathrm{~K})$, (triangles); (b) significant diffusive leakage through the cladding layers ( $T=400 \mathrm{~K}$ ) (squares), and (c) significant drift and diffusive leakage (400 K) (circles).

$P_{\text {ext }}(I)$ curves $\left(\eta_{\text {ext }}^{d}\right)$ at a specified current relative to threshold for modeled devices of different length. Values of $\alpha_{i}$ were then determined from $1 / \eta_{\text {ext }}^{d}$-length plots using Eq. (1), and the process was repeated for sets of values for $\eta_{\text {ext }}^{d}$ obtained at different currents above threshold. This produces the data in Fig. 2 for the value of $\alpha_{i}$ derived at different currents on the light-current curve. The data shown as triangles were modeled at $300 \mathrm{~K}$ corresponding to the situation in Fig. 1 where $J_{L}=0$, and shows that once the Fermi levels have pinned, $\left(I>1.3 I_{\mathrm{th}}\right)$ the derived value of $\alpha_{i}$ agrees with the input value of $10 \mathrm{~cm}^{-1}$.

To examine the effect of carrier leakage through the cladding layers we repeated the modeling at $400 \mathrm{~K}$, where these effects are significant, for two cases: leakage by diffusion only, and leakage by diffusion and drift. The former was done by setting $z=\infty$ in Eq. (5) and leads to the derived values for $\alpha_{i}$ plotted in Fig. 2 as squares. Again for currents above $1.3 I_{\text {th }}$ the input value of $10 \mathrm{~cm}^{-1}$ is obtained and the effect of diffusion current is simply to increase the error where the Fermi levels do not saturate. Since the diffusion current is controlled by the Fermi levels at the edges of the cladding layers, once the Fermi levels pin these currents also saturate.

With leakage by both diffusion and drift included in the model we derived the values of $\alpha_{i}$ shown by the circles in Fig. 2. Above $1.3 I_{\text {th }}$ these values become independent of the current at which the measurement is made, nevertheless they are about half the input value. This is because, although the Fermi levels clamp at the cladding layer edge, the drift current continues to increase due to the increase in field across the cladding caused by the increasing majority carrier current, as indicated by Eq. (6). In this case the Fermi levels are not pinned throughout the whole of the current carrying region and the injection efficiency varies with cavity length, as therefore does $\eta_{0}^{d}$, leading to significant error in the derived value of mode loss. Under these conditions the analysis is clearly inappropriate.

We have investigated the validity of the analysis to obtain $\alpha_{i}$ by means of a rate equation model coupled to models 
for spontaneous and nonradiative recombination in the well and carrier leakage by drift and diffusion through the cladding layers. We conclude that:

(i)

because the Fermi levels do not pin instantly at threshold the external efficiency must be evaluated at sufficiently high current that the $P-I$ curve is linear ( $\eta_{\text {ext }}^{d}$, is constant with current) and $\eta_{r}^{d}=1$ (the minimum acceptable current depends upon the value of $\left.\beta_{\text {spon }}\right)$;

(ii) even in the presence of purely diffusive leakage a reliable value for the loss is obtained provided the measurement is made over the correct current range; but

(iii) in the presence of leakage by drift the derived value of loss is seriously in error because the Fermi levels are not pinned throughout the whole structure and the internal efficiency itself is length and temperature dependent.
The calculations including leakage have been done at $400 \mathrm{~K}$ to accentuate the effect, however the key point is that the conventional analysis is inappropriate where drift leakage is significant, no matter what the temperature. The experimental criteria for a valid measurement are that the external differential efficiency should be independent of current level above threshold and independent of temperature.

${ }^{1}$ J. R. Biard, W. N. Carr, and B. S. Reed, Trans. AIME 230, 286 (1964).

${ }^{2}$ P. A. Andrekson, N. A. Olsson, T. Tanbun-Ek, R. A. Logan, D. Coblentz, and H. Temkin, Electron. Lett. 28, 171 (1992).

${ }^{3}$ P. M. Smowton and P. Blood, Appl. Phys. Lett. 70, 1073 (1997).

${ }^{4}$ G. P. Agrawal, and N. K. Dutta, Semiconductor Lasers (Van Nostrand Reinhold, New York, 1993).

${ }^{5}$ P. M. Smowton and P. Blood, "Visible emitting (AlGa)InP laser diodes," in Strained Quantum Wells and their Applications, edited by M. O. Manasreh (Gordon and Breach, New York, in press).

${ }^{6}$ D. P. Bour, D. W. Treat, R. L. Thornton, R. S. Geels, and D. F. Welch, IEEE J. Quantum Electron. 29, 1337 (1993).

${ }^{7}$ P. M. Smowton, H. D. Summers, P. Rees, and P. Blood, IEE Proc. Optoelectronics 141, 136 (1994). 